\title{
DESCRIPTION OF A WEARABLE ELECTROENCEPHALOGRAPHY + FUNCTIONAL NEAR- INFRARED SPECTROSCOPY (EEG+FNIRS) FOR IN-SITU EXPERIMENTS ON DESIGN COGNITION
}

\author{
Dybvik, Henrikke; \\ Kuster Erichsen, Christian; \\ Steinert, Martin \\ Norwegian University of Science and Technology
}

\begin{abstract}
We developed a wearable experimental sensor setup featuring multimodal EEG+fNIRS neuroimaging data capture applicable for in situ experiments at a lower financial threshold. Consistent application of a good protocol and procedure for sensor application and signal quality control is crucial for researchers to obtain valid data. This paper provides an exhaustive description of the sensor setup, the data synchronization process, procedure for sensor application, and signal quality control. Potential design cognition experiments with the proposed EEG+fNIRS are also described. In summary, the setup is mobile and provides multimodal neuroimaging data of high quality. We encourage the design community to take advantage of the setup and adapt it to new experimental setups in situ.
\end{abstract}

Keywords: EEG+fNIRS, Mobile Experiments, Human behaviour in design, Design cognition, Research methodologies and methods

\section{Contact:}

Dybvik, Henrikke

Norwegian University of Science and Technology

Department of Mechanical and Industrial Engineering

Norway

henrikke.dybvik@ntnu.no

Cite this article: Dybvik, H., Kuster Erichsen, C., Steinert, M. (2021) 'Description of a Wearable

Electroencephalography + Functional Near-Infrared Spectroscopy (EEG+FNIRS) for In-Situ Experiments on Design Cognition', in Proceedings of the International Conference on Engineering Design (ICED21), Gothenburg, Sweden, 16-20 August 2021. DOI:10.1017/pds.2021.94 


\section{INTRODUCTION}

Interaction design, human-centred design (HCD) and human-computer interaction (HCI) use experiments for many purposes. To evaluate user interfaces and styles of interaction; understand how people use, experience, perceive and process interactive and increasingly complex technology; and to further understand the underlying mechanisms of human cognition in interaction with objects and technical systems (Balters \& Steinert, 2017; Blessing \& Chakrabarti, 2009; Cairns \& Cox, 2008). Information gathered through neuroimaging techniques and physiology sensors can provide knowledge of mental state and cognition which benefit interface design and product development both in the early design process and at the evaluation stage, for new and existing systems (Balters \& Steinert, 2017; Cairns \& Cox, 2008; Wulvik et al., 2019). On a methodological level, design research is increasing experiments with physiology and neuroimaging measurements (Balters \& Steinert, 2017; Goucher-Lambert et al., 2019; Hay et al., 2020; Steinert \& Jablokow, 2013). Such experiments often exist with a trade-off between experimental control and ecological validity (Hay et al., 2020), which is problematic since laboratory settings simply doesn't produce results replicable in the real word (Cairns \& Cox, 2008; Okamoto et al., 2004). Highly ecologically valid (aka in situ) studies demonstrate how humans appropriate technological solutions in their intended context. Such studies accommodate the often unpredictable, real-world environments in which technology is used (Consolvo et al., 2007). Thus, they are suited to study engineering design solutions and activities (Balters \& Steinert, 2017; Hay et al., 2020; Mayseless et al., 2019). Electroencephalography (EEG) and functional near-infrared spectroscopy (fNIRS) are two portable sensor modalities serving as a substitute for neuroscience gold standard fMRI. EEG (electrical brain activity) and fNIRS (cerebral hemodynamic response) are complementing techniques that don't strictly immobilize participants (Herold et al., 2018; Jacko, 2012; Pinti et al., 2018). Neuroimaging modalities may best be used in combination with other modalities, such as systemic measurements (physiology sensors such as electrocardiography and galvanic skin response) and behavior (eye tracking, motion capture, video recordings) (Pinti et al., 2018; Xu et al., 2019), because it enables data and method triangulation. Triangulation increase validity of results, reduce bias and error, and accommodate individualism (i.e. individual behavior, physiology and psychology) (Balters \& Steinert, 2017; Blessing \& Chakrabarti, 2009; Steinert \& Jablokow, 2013). Multimodal EEG+fNIRS is used in cognitive neuroscience, design research, HCI research, allowing investigation of cognitive states (Ahn et al., 2016; Ahn \& Jun, 2017; Hassib et al., 2017; Jacko, 2012; Lukanov et al., 2016; Mayseless et al., 2019; Pinti et al., 2018). EEG+fNIRS are now not only portable, but becoming increasingly wearable (Hassib et al., 2017; Pinti et al., 2018; Xu et al., 2019), thus suited for in situ studies. However, portable neuroimaging systems are expensive compared to other portable physiology measures (Cisler et al., 2019). Moreover, development of wearable and wireless technology usually come with the cost of low resolution (Piper et al., 2014) and there are limited options for multimodal data capture systems (Ahn \& Jun, 2017). Thus, there is a need for a wearable experimental sensor-setup featuring multimodal neuroimaging with high resolution data capture, applicable for in situ studies, at a lower financial threshold.

\subsection{The study goal}

The goal of this work is to develop a low-cost wearable neuroimaging sensor setup, consisting of concurrent EEG and fNIRS measurements. A wearable fNIRS system was integrated with a low-cost EEG sensor, and a data synchronization process established. This paper contributes an exhaustive description of the sensor setup, and detail the procedure for sensor application, and protocol for signal quality control which is needed to ensure collection of high-quality data.

The second section provides theoretical background on EEG and fNIRS. Then, the functional prototype is presented in the third section. Discussion follows in section five, before the conclusion in section six.

\section{BACKGROUND OF EEG AND FNIRS}

\subsection{Technical principle of EEG}

Electroencephalography (EEG) is a neuroimaging tool measuring electrical brain activity, specifically the electric field caused by local current flow induced by neural activation (Malmivuo \& Plonsey, 1995). Neural activation generates an electrical potential difference measured by a pair of electrodes mounted on the scalp surface. An EEG signal consists of the sum of neural activity in close vicinity to 
the electrodes (Balters \& Steinert, 2017; Herold et al., 2018). The signal can be recorded inside the skull (intracranial), or on the scalp surface (extracranial) (Im, 2018; Malmivuo \& Plonsey, 1995). Intracranial EEG is not relevant for this work. Signal magnitude is in the range of $\pm 100 \mu \mathrm{V}$ and the frequency spectrum is conventionally considered to be in the range of $0-100 \mathrm{~Hz}(\mathrm{Im}, 2018$; Malmivuo \& Plonsey, 1995). EEG signals need to be amplified and converted from analogue to digital before processing and analysis (EEG has low signal-to-noise ratio due to the skulls' inherent low conductivity (Balters \& Steinert, 2017; Teplan, 2002). Many different electrodes with different characteristics exists (Lee et al., 2019; Teplan, 2002). The international EEG system provides a standardized electrode positioning system, enabling greater replicability of EEG studies (Oostenveld \& Praamstra, 2001).

\subsection{Technical principle of fNIRS}

Functional Near-Infrared Spectroscopy (fNIRS) is a neuroimaging technique that optically measures the hemodynamic response in brain tissue. Concentration changes in oxygenated and deoxygenated hemoglobin $\left(\Delta \mathrm{HbO}_{2}\right.$ and $\Delta \mathrm{HbR}$ respectively) are related to neural activation (Pinti et al., 2018; Xu et al., 2019). Light of different wavelengths in the near-infrared (NIR) spectrum is emitted on the scalp, travels through different layers of the head (e.g., scalp skin, skull, cerebrospinal fluid) before reaching neuronal tissue. Inside the tissue NIR light undergoes absorption and scattering, each contributing to light attenuation. Hemoglobin absorbs NIR light differently depending on oxygen saturation state, which changes light attenuation. Non-absorbed light scatter components are measured and $\Delta \mathrm{HbO}_{2}$ and $\Delta$ HbR calculated through the modified Beer-Lambert Law (Herold et al., 2018; Pinti et al., 2018). Two or more optodes, i.e., a minimum of one source and one detector must be used. Active brain regions are generally associated with increased $\Delta \mathrm{HbO}_{2}$ and decreased $\triangle \mathrm{HbR}$. The international EEG system (Oostenveld \& Praamstra, 2001) is often used for optode placement, but there are works where this is not the case. An interoptode distance of $3 \mathrm{~cm}$ is common (Herold et al., 2018).

\subsection{Advantages and limitations}

EEG gained popularity due to being non-invasive, portable, having high temporal resolution, and being relatively inexpensive compared to other neuroimaging techniques (Balters \& Steinert, 2017; Pinti et al., 2018; Solovey et al., 2009). However, EEG limitations include low spatial resolution, proneness to noise from motion, artifacts due to sweat and/or muscle activity (Al-Shargie et al., 2016; Balters \& Steinert, 2017; Pinti et al., 2018). fNIRS offers better spatial resolution, is more robust to motion artifacts from head and body, but has lower temporal resolution (Al-Shargie et al., 2016; Herold et al., 2018; Pinti et al., 2018). fNIRS furthermore share several advantages with EEG, notably non-invasiveness, portability, being relatively lightweight, compact, and low-cost. Both are silent in operation, safe for longer term measurement and suitable for multimodal imaging (Al-Shargie et al., 2016; Lee et al., 2019; Pinti et al., 2018; Solovey et al., 2009). fNIRS is limited to cortical layers of the brain (Herold et al., 2018; Jacko, 2012; Pinti et al., 2018). Due to the nature of the hemodynamic response, a $\sim 5$ second delay from stimuli onset to peak response is inherent in fNIRS data (Pinti et al., 2018), however, EEG measures electrical responses instantly. fNIRS measurements are sensitive to changes in scalp blood flow unrelated to brain activation, and changes in systemic physiology (e.g. increase in heart rate) (Herold et al., 2018; Jacko, 2012; Pinti et al., 2018), and ambient light can contribute to noisy data (Solovey et al., 2009), but this is not the case for EEG. EEG and fNIRS are complementing techniques and provide much of the information obtainable by neuroscience gold standard fMRI, while rendering strict immobilization of participants unnecessary (Herold et al., 2018; Jacko, 2012).

\section{SETUP DESCRIPTION}

This section describes the wearable EEG+fNIRS sensor system and include hardware, sensor integration, software for data collection, and process for ensuring high data quality.

\subsection{Physical setup}

The physical setup consists of a core setup (Fig. 1.) with possibilities for additional physiological measurements and devices.

Core setup:

- Laptop (Dell Latitude 7490)

- fNIRS (NIRSport (NIRSport, 2015), with 8+8 optodes) 
- EEG (Open BCI (OpenBCI Inc., 2019), with 8 dry electrodes)

- EEG adapter (Erichsen et al., 2020)

- Flexible fabric cap (128Ch Standard Cap for NIRX, EASYCAP GmbH, Germany) Additional equipment for a conventional computer setup:

- Monitor, keyboard, mouse, web camera.

- HDMI cable, USB cables, USB port hub
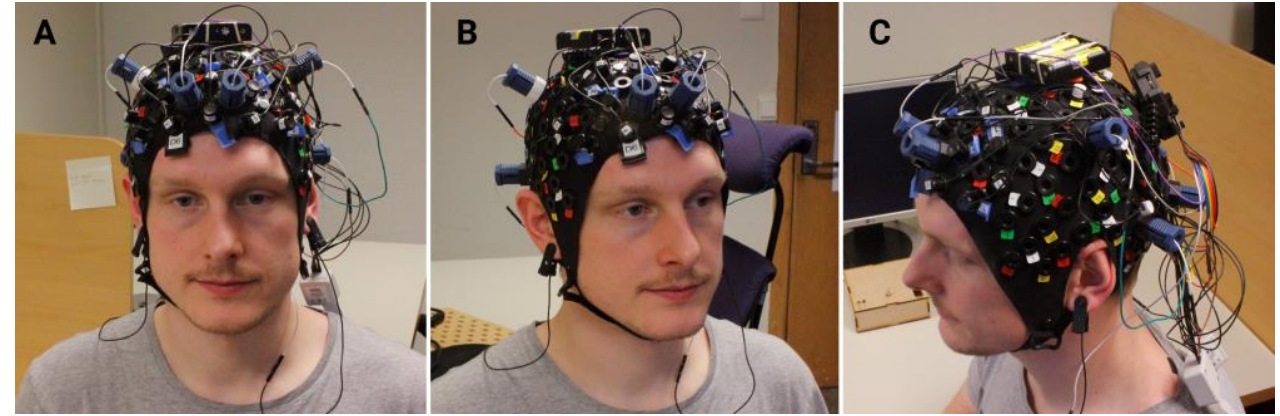

Figure 1. The cap fully integrated with fNIRS and EEG adapters. A) Battery pack placed on the head. B) EEG adapters protrude more than optodes. C) Cables are led toward the back and secured with clips to be of minimal disturbance for the participant. The participant provided written consented to appear in the images published.

\subsection{Detailed sensor description of core setup}

For collection of fNIRS data the NIRSport system (NIRSport, 2015) with 8 sources and 8 detectors was used with two wavelengths $(760 \mathrm{~nm}$ and $850 \mathrm{~nm})$ sampled at $7.81 \mathrm{~Hz}$. For collection of EEG data we used the Cyton biosensing board from OpenBCI (OpenBCI Inc., 2019), with 8 spring-loaded, dry electrodes provided as part of the Ultracortex Mark IV EEG headset (Ultracortex "Mark IV" EEG Headset, 2019) sampled at $250 \mathrm{~Hz}$. Both fNIRS optodes and EEG electrodes were mounted on a '128Ch Standard Cap (EASYCAP GmbH, Germany), that follows the five percent system (Oostenveld \& Praamstra, 2001). EEG was mounted with the adapter described in the following section (3.3). A dedicated Dell Latitude 7490 laptop was used to collect data. It runs Microsoft Windows 10 Education with a Intel(R) Core(TM) i7-8650U CPU @ 1.90GHz 2.11 GHz processor, 32.0 GB RAM installed, 64-bit operating system, x64-based processor and a 500 GB SSD hard drive.

\subsection{Sensor integration in one cap}

fNIRS optodes and EEG electrodes were mounted on a standard cap. Initially, this cap only accommodates more expensive EEG electrodes with a geometrical form similar to the fNIRS optodes, and not the selected spring-loaded dry electrodes from OpenBCI. To integrate EEG with fNIRS, we developed a novel adapter that interface between EEG electrodes and the standard cap. The adapter consists of two octagonal parts with a circular, central hole allowing access to the scalp. The adapter is mounted on the cap in the following manner: The bottom part is positioned on the inside of the cap and extends through the precut holes and secured to the top part by means of a threaded connection. The spring-loaded electrode-mounts from OpenBCI are attached to the adapter by a threaded connection, illustrated in Fig. 2. Existing fNIRS optode holders are used to fasten the Cyton board and battery to the participant's head (rendered possible by the small size and low weight ( 160 gram) of the battery and board). This imposes fewer restrictions on movement. If the desired optode or electrode configuration conflicts with placement of the battery, the battery can be mounted to the participant's body by elongating the wires from the battery pack. These adapters allow the springloaded, dry electrodes to be mounted in any of the 128 standardized positions on the cap that are not occupied by fNIRS optodes. This drastically increases the flexibility and accuracy in electrode placement compared to currently available low-cost EEG equipment e.g., from Emotiv, Neurosky, InteraXon, as well as the standard solutions from OpenBCI. This approach has three advantages. Firstly, the increased flexibility in electrode placement provides more control over which brain regions to study and thus extends possible use cases for OpenBCI hardware. Secondly, accurate electrode positioning is essential in EEG research to ensure replicability and compare results across studies (Oostenveld \& Praamstra, 2001). Thus, the approach increases the viability of applying a low-cost 
EEG device in scientific research. The complete setup enables low-cost integration of EEG with fNIRS for multimodal brain imaging, lowering the financial barrier to utilize this cutting-edge research method. An exhaustive description of adapter development can be found in Erichsen et al. (2020), and production files at https://github.com/trolllabs/eeg-technology.
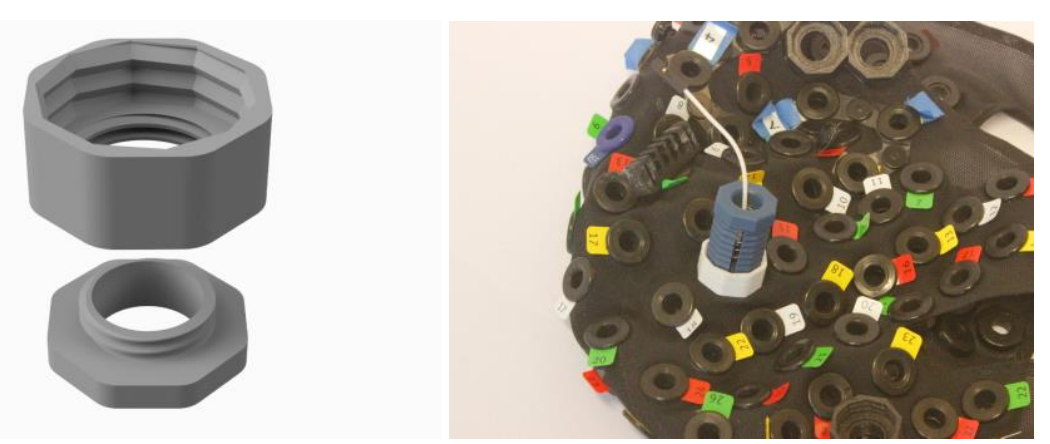

Figure 2. Adapter for EEG-integration. A) Adapter developed to interface the spring-loaded electrodes from OpenBCl with the fNIRS electrode cap. B) Spring loaded electrode mounted on the cap using the adapter.

\subsection{Software securing data collection}

iMotions 8.1 platform (The IMotions Platform, 2020) present stimuli, collect and synchronize all sensor data. iMotions offer the possibility of registering one or more Lab Streaming Layer (LSL) streams as a sensor. LSL is "a system for the unified collection of measurement time series in research experiments that handles both the networking, time-synchronization, (near-) real-time access as well as optionally the centralized collection, viewing and disk recording of the data"1. Nirstar 15.2 Acquisition Software for NIRSport (NIRSport, 2015) allows preview, recording and forwarding fNIRS data to third-party software. NIRStar 15.2's LSL option was enabled, and preview mode used to forward a continuous fNIRS data stream through LSL. A python script interfacing with LSL detects the fNIRS data stream and forwards these to iMotions API integration. This enables continuous data collection of all fNIRS channels. For EEG, a separate Python script detects the EEG stream and forwards the data to iMotions using LSL.

\subsection{Process for ensuring high signal and data quality}

Consistency in experimental procedures ensures that every participant has the same experience, and limits confounding variables and aid experiment robustness (Cairns \& Cox, 2008). The current procedure includes greeting participants, acquiring informed consent, a short briefing, placing sensors, ensure connectivity and signal quality, before starting the experiment. Variability in required setup time can occur due to difficulties obtaining good signal quality from both fNIRS and EEG simultaneously. Both optodes and electrodes require direct scalp contact, which is affected by amount and thickness of hair, the ease of which hair can be parted, and general skin conductance. The need to manually disperse hair, apply optically or electrically conductive gels, or spend extensive time organizing wires can significantly impact setup time. The consistent application of a good protocol and procedure for sensor application and signal quality control is crucial for researchers to obtain valid data that can be for inference making. Given this importance the following details steps toward obtaining high signal quality, including an electrode/optode placement procedure and visual inspection prior to starting data collection.

\subsubsection{Protocol for ensuring data quality in EEG}

Electrode placement procedure. Since fNIRS system current can cause electrical inference and distort EEG-signals (Lee et al., 2019), a wire-configuration that minimize electrical inference is crucial. Several strategies minimize crosstalk; 1) Ensure good electrical contact between electrodes and scalp, since poor electrical contact increase EEGs susceptibility to noise. 2) Maximizing distance between EEG and fNIRS leads. 3) Ensure electric isolation between, and separate ground planes for

1 https://github.com/sccn/labstreaminglayer/ Accessed 13.04.20 
fNIRS and EEG. Obtaining proper spacing can be challenging and the difficulty increase with increasing number of optodes and electrodes. The dry EEG electrode's conductive coating is susceptible to mechanical wear, which limits operational life to 20-30 uses. To limit mechanical wear, electrodes should be mounted after the cap is correctly positioned on the head. Hair in electrode locations should be parted (with a wooden applicator, a Q-tip or similar) to facilitate direct contact with the scalp. Preferably the scalp should be visible through the hole in the cap. The spring-loaded electrodes are then inserted into adapters and fastened by clockwise rotation. Electrodes must be able to move freely in the housing. Excessively fastened electrodes negate the function of the spring loading. Therefore, minimizing hair in areas where electrodes are to be mounted is essential. Applying some conducting gel or water solution to increase the conductivity in hairy regions might be advantageous. However, this can introduce drawbacks associated with wet electrodes such as evaporation of the conducting compound and the need to clean equipment after use. This may also limit the possible duration of the recording.

Visual inspection of signal quality before recording. OpenBCI GUI (open-source software) was used to visually inspect EEG data and signal quality. This was necessary since iMotions is not suited to display real-time EEG-data. OpenBCI GUI allows inspection of data in numerous forms, including a live time-series plot and an FFT-plot indicating the frequency distribution for each channel. Impedance for each channel can also be measured. The FFT-plot should indicate gradually decreasing power towards higher frequencies. fNIRS crosstalk will produce peaks in the FFT-plot at harmonics of the fNIRS sampling rate $(7.81 \mathrm{~Hz})$, indicative of excessive noise in the signal, and is to be avoided. Very poor electrical contact between electrodes and scalp is indicated by "Near railed" or "Railed" in the time-series plot.

\subsubsection{Protocol for ensuring high data quality in fNIRS}

Optode placement procedure. For best signal quality ensure a low-loss contact between skin and optodes. Care should be taken to stabilize optodes on the skin to prevent signal variations and artifacts from motion. The hair should be properly parted, preferably clearly seeing the scalp. A wooden applicator can be used to part hair before placing optodes if needed. If participants have particularly dense hair use of a nonconductive clear gel is recommended, since it improves optical contact and can make parting hair easier. A light source can be helpful in this process. Additional steps to ensure a stable optode can be taken; 1) The use of a stiffening joint increase mechanical rigidity of the montage and facilitate equal optode distance. 2) Fiber optic cables can be spatially organized in a cable tree to avoid undue torque and tipping of optodes. 3) A retaining overcap can be used to exert even pressure on probes and stabilize setup against motion. This overcap also block external light sources from affecting the NIR-light. This was not possible due to the EEG integration, which are larger in size, and the standardized overcap would either distort the signal or destroy the adaptive holder.

Visual inspection of signal quality before recording. NIRStar 15.2 's preview mode was used to visually inspect fNIRS data and signal quality. First, a calibration must be performed, and the results analyzed to control signal quality. A Quality Scale tool in NIRStar composes several parameters affecting signal quality: the gain levels, the signal level, the noise level, and a hemodynamic index. A color scale of green, yellow, and red illustrate signal quality collectively. All channels should be green, however yellow may also be accepted. The parameters can be viewed and inspected individually, which should be performed if channels are not acceptable (red or yellow). Gain and intensity of received light have an inverse relationship, if a signal is weak it must be amplified more, which increase noise levels. Noise can be computed as a Coefficient of Variation (CV), indicating variability of the signal with respect to its mean value. Channels with $C V>15 \%$ will most likely not yield good signal quality. Viewing parameters individually gives a better understanding of why poor signal quality occurs and may help when trying to improve signal quality. High signal quality is generally indicated by visible oscillations of approximately $1 \mathrm{~Hz}$ (corresponding to the cardiac response), and overall smooth signal curve. Abrupt spikes do not correspond to the hemodynamic response, they could indicate motion artifacts or noise and should not be visible. Motion artifacts should not be visible if the cap is set up properly, which could be tested by the participant moving their head, in which case the signal should not deteriorate. 


\section{DISCUSSION AND FUTURE WORK}

\subsection{Flexibility and robustness}

The EEG adapter interface with the spring-loaded electrode mount from OpenBCI, which is compatible with three different dry electrode geometries: flat, spikey, and $5 \mathrm{~mm}$ combs. We applied $5 \mathrm{~mm}$ comb electrodes in hair-covered regions $(\mathrm{C} 3, \mathrm{C} 4, \mathrm{P} 7, \mathrm{P} 8, \mathrm{O} 1, \mathrm{O} 2)$ and spikey electrodes in hairless locations (AFp1 and AFp2). Electrodes can be placed in any position, except for positions occupied by fNIRS. Should different EEG positions be selected or should hair density/conditions vary across participants, electrode geometry can be selected to facilitate scalp contact. We can select different battery packs and different 3D printing filament, which in turn affects both price and robustness. The battery pack can be relocated to a different body part. To reduce electrical inference from fNIRS in EEG signals various shielding measures were tested, but results did not indicate any obvious effects on electrical inference. We found best signal quality when ensuring maximum distance between fNIRS wires and EEG leads. We settled with shielding reference wires and placed fNIRS wires close to the face but will continue testing additional shielding measures to improve setup robustness.

We do not consider data synchronization to be completely robust yet. The iMotions platform do synchronize data, but we have had experiences with occasional unexpected crashes of the platform. Moreover, the platform is demanding for the computer. Our recommendations for those that may be interested in acquiring a similar setup is to investigate open-source software. Our experience with open source software, notably using LSL has been good so far, and we see other studies also using it (Cisler et al., 2019). We will continue developing our software setup by further utilizing LSL, investigating its use in combination with stimuli presentation tools suited to our experiment designs and research questions, and we encourage the community to do the same. Furthermore, not all studies incorporate concurrent physiological measurements disclose exactly how data was synchronized (Liu et al., 2017) and since we are interested in best practice of data synchronization, we highly encourage other studies to disclose their data synchronization process.

\subsection{Portability}

All individual components are portable. EEG stream data wirelessly over Bluetooth to the laptop. fNIRS requires a cabled connection to the amplifier and can be extended up to $3 \mathrm{~m}$. It can still be applied in experiments involving a moderate amount of movement. The setup can be operated completely on battery power, although only for a limited time. EEG and fNIRS each have dedicated batteries; fNIRS lasts 8 hours; EEG is power by 4 AA alkaline batteries, which lasts at least as long as fNIRS. The longest recording conducted completely on battery power lasted 66 minutes and collected EEG, fNIRS and one web camera recording. This was limited by laptop battery. If the laptop receives socket power, or uses a power bank, this is no longer the limiting factor. The minimum necessary hardware components to conduct an in situ study include EEG electrodes and fNIRS optodes, their respective amplifiers and battery, and a laptop. This entire configuration can be put in a normal backpack. The setup has been relocated several times, from offices, to research laboratory and different apartments for testing. Another paper (Dybvik et al., 2021) demonstrate use in a car while driving and a yoga practice, which required transportation to, and set up in a car and a living room. Considering these characteristics in combination with these use cases we pose the setup a viable tool for capturing physiological responses during in situ experiments.

\subsection{Cost}

The costs associated with the EEG integration includes $\$ 849$ for the hardware supplied by OpenBCI (excluding shipping), and roughly $\$ 5$ in filament cost for printing adapters, assuming free access to a 3D-printer. The dry electrodes and ear clips must be replaced after 20-30 uses since the conductive coating wear off. Replacement electrodes can be acquired for a cost as low as $\$ 0.50 /$ pcs depending on volume. We regard this as low-cost when compared to the price of other wearable EEG systems we're aware of, e.g., \$ 26500 for BIOPAC bioharness and BIOPAC B-Alert System. Since wearable fNIRS systems (with more than 1 channel) amount to $€ 40000$ or more, cost associated with concurrent EEG+fNIRS quickly aggregates, and we hope the EEG adaptation can be a low-cost option for the community. 


\subsection{Comparing our setup with existing setups}

Current high resolution multimodal EEG+fNIRS systems either require two desktop computers (Ahn et al., 2016; Ahn \& Jun, 2017), or two separate instrument systems (Al-Shargie et al., 2016; Shin et al., 2017), each not portable and at a significant cost. Despite fNIRS' and EEGs general increase in portability (Hassib et al., 2017; Xu et al., 2019) and potential for wireless data transmission (Pinti et al., 2018; von Lühmann et al., 2015), wireless fNIRS is not the norm. Moreover, many of the most portable technologies collect data at the lowest resolution possible, which is single channel measurements for both EEG and fNIRS (Xu et al., 2019). This naturally contributes to low weight, less expenses, and easier use in situ since preparation time reduces with the number of channels, but this highly limits brain regions and constructs of interest. Researchers have built wireless EEG+fNIRS, developing open source systems with up to 4+2 channel EEG and fNIRS measurements (von Lühmann et al., 2015, 2017). One system support 32 sources and 4 detectors, and up to 16 electrodes, but require use of gel (Safaie et al., 2013). Another portable system support 8 EEG and 32 fNIRS channels, but require a control module to be carried around with the participant (Sawan et al., 2013), similar to our setup. However, we are unable to find participant studies using any of the mentioned systems. We speculate system reproduction might be too time-consuming for practical purposes. Our setup resolution is 8 EEG electrodes and $8+8$ fNIRS optodes. Regarding data quality, the EEG OpenBCI Cyton board exhibits comparable quality as medical-grade equipment EEG (Frey, 2016), whereas NIRx dedicate their equipment for scientific applications. One limitation of our setup is that we cannot collect concurrent EEG and fNIRS measurements from the same location, but this also applies for other setups.

\subsection{Proposed examples of usage}

Neuroimaging have been used to investigate designers cognitive style in divergent and convergent design problems (Steinert \& Jablokow, 2013), the effectiveness of inspirational stimuli (GoucherLambert et al., 2019), and the difference in cognition associated with problem-solving and open-ended design tasks (Vieira, Gero, Delmoral, Gattol, et al., 2020; Vieira, Gero, Delmoral, Li, et al., 2020). We now know that brainstorming and TRIZ evoke different cognitive processes (Shealy et al., 2020), and begin to understand the role of stress during conceptual design (Nguyen \& Zeng, 2014). There are numerous opportunities in design cognition, e.g. how different types of memory and knowledge are used in design tasks, the general effects of stimuli on creativity, and how higher-order processing operates in problem-solution coevolution (Hay et al., 2020). We think these topics are interesting and suggest them as potential topics for other researchers to investigate with the proposed EEG+fNIRS setup.

\subsection{Further work}

Further work includes improving data capture and synchronization, first by further utilizing LSL. We both expect and experience increased noise and motion artifacts in situ, and we will investigate means to further minimize noise, by testing and learning from hardware development mentioned (Safaie et al., 2013; Sawan et al., 2013; von Lühmann et al., 2015, 2017). Shielding and wire layout will be improved to minimize crosstalk. In situ testing will continue and improvements made to the setup to advance its robustness and to further reduce setup reproduction time.

\section{CONCLUSION}

This paper presents a wearable experimental sensor setup featuring multimodal EEG+fNIRS neuroimaging data capture applicable for in situ experiments. A low-cost EEG was integrated with a wearable fNIRS system, for which we provide an exhaustive description of sensor setup, data synchronization process, procedure for sensor application, and signal quality control. An EEG+fNIRS sensor setup is both applicable in and valuable for e.g., design cognition research. We will continue to develop the setup and conduct experiments. We encourage the community to do the same: take advantage of the setup and adapt it to your in situ experiments.

\section{ACKNOWLEDGMENTS}

The study was approved by the Norwegian Centre for Research (NSD). This research is supported by strategic funds from the department for Mechanical and Industrial Engineering (MTP) at NTNU. 


\section{REFERENCES}

Ahn, S., \& Jun, S. C. (2017). Multi-Modal Integration of EEG-fNIRS for Brain-Computer Interfaces - Current Limitations and Future Directions. Frontiers in Human Neuroscience, 11. https://doi.org/10.3389/fnhum.2017.00503

Ahn, S., Nguyen, T., Jang, H., Kim, J. G., \& Jun, S. C. (2016). Exploring Neuro-Physiological Correlates of Drivers' Mental Fatigue Caused by Sleep Deprivation Using Simultaneous EEG, ECG, and fNIRS Data. Frontiers in Human Neuroscience, 10. https://doi.org/10.3389/fnhum.2016.00219

Al-Shargie, F., Kiguchi, M., Badruddin, N., Dass, S. C., Hani, A. F. M., \& Tang, T. B. (2016). Mental stress assessment using simultaneous measurement of EEG and fNIRS. Biomedical Optics Express, 7(10), 3882. https://doi.org/10.1364/BOE.7.003882

Balters, S., \& Steinert, M. (2017). Capturing emotion reactivity through physiology measurement as a foundation for affective engineering in engineering design science and engineering practices. Journal of Intelligent Manufacturing, 28(7), 1585-1607. https://doi.org/10.1007/s10845-015-1145-2

Blessing, L. T., \& Chakrabarti, A. (2009). DRM, a design research methodology. Springer Science \& Business Media.

Cairns, P. E., \& Cox, A. L. (2008). Research methods for human-computer interaction. Cambridge University Press.

Cisler, D., Greenwood, P. M., Roberts, D. M., McKendrick, R., \& Baldwin, C. L. (2019). Comparing the Relative Strengths of EEG and Low-Cost Physiological Devices in Modeling Attention Allocation in Semiautonomous Vehicles. Frontiers in Human Neuroscience, 13. https://doi.org/10.3389/fnhum.2019.00109

Consolvo, S., Harrison, B., Smith, I., Chen, M. Y., Everitt, K., Froehlich, J., \& Landay, J. A. (2007). Conducting In Situ Evaluations for and With Ubiquitous Computing Technologies. International Journal of HumanComputer Interaction, 22(1-2), 103-118. https://doi.org/10.1080/10447310709336957

Dybvik, H., Erichsen, C. K., \& Steinert, M. (2021). Demonstrating the feasibility of multimodal neuroimaging data capture with a wearable electroencephalography + functional near-infrared spectroscopy (EEG+FNIRS) IN SITU. Proceedings of the Design Society: International Conference on Engineering Design.

Erichsen, C. K., Dybvik, H., \& Steinert, M. (2020). Integration of low-cost, dry-comb EEG-electrodes with a standard electrode cap for multimodal signal acquisition during human experiments. DS 101: Proceedings of NordDesign 2020, Lyngby, Denmark, 12th - 14th August 2020, 1-12. https://doi.org/10.35199/NORDDESIGN2020.19

Frey, J. (2016, May 30). Comparison of a consumer grade EEG amplifier with medical grade equipment in BCI applications. International BCI meeting. https://hal.inria.fr/hal-01278245

Goucher-Lambert, K., Moss, J., \& Cagan, J. (2019). A neuroimaging investigation of design ideation with and without inspirational stimuli-Understanding the meaning of near and far stimuli. Design Studies, 60, 1-38. https://doi.org/10.1016/j.destud.2018.07.001

Hassib, M., Schneegass, S., Eiglsperger, P., Henze, N., Schmidt, A., \& Alt, F. (2017). EngageMeter: A System for Implicit Audience Engagement Sensing Using Electroencephalography. Proceedings of the $2017 \mathrm{CHI}$ Conference on Human Factors in Computing Systems, 5114-5119. https://doi.org/10.1145/3025453.3025669

Hay, L., Cash, P., \& McKilligan, S. (2020). The future of design cognition analysis. Design Science, 6. https://doi.org/10.1017/dsj.2020.20

Herold, F., Wiegel, P., Scholkmann, F., \& Müller, N. G. (2018). Applications of Functional Near-Infrared Spectroscopy (fNIRS) Neuroimaging in Exercise-Cognition Science: A Systematic, Methodology-Focused Review. Journal of Clinical Medicine, 7(12), 466. https://doi.org/10.3390/jcm7120466

Im, C.-H. (Ed.). (2018). Computational EEG Analysis: Methods and Applications. Springer Singapore. https://doi.org/10.1007/978-981-13-0908-3

Jacko, J. A. (2012). The human-computer interaction handbook: Fundamentals, evolving technologies, and emerging applications (3rd ed.). CRC Press.

Lee, S., Shin, Y., Kumar, A. R., Kim, M., \& Lee, H. (2019). Dry Electrode-Based Fully Isolated EEG/fNIRS Hybrid Brain-Monitoring System. IEEE Transactions on Biomedical Engineering, 66, 1055-1068. https://doi.org/10.1109/tbme.2018.2866550

Liu, Y., Ayaz, H., \& Shewokis, P. A. (2017). Multisubject "Learning” for Mental Workload Classification Using Concurrent EEG, fNIRS, and Physiological Measures. Frontiers in Human Neuroscience, 11. https://doi.org/10.3389/fnhum.2017.00389

Lukanov, K., Maior, H. A., \& Wilson, M. L. (2016). Using fNIRS in Usability Testing: Understanding the Effect of Web Form Layout on Mental Workload. Proceedings of the 2016 CHI Conference on Human Factors in Computing Systems, 4011-4016. https://doi.org/10.1145/2858036.2858236

Malmivuo, J., \& Plonsey, R. (1995). Bioelectromagnetism: Principles and Applications of Bioelectric and Biomagnetic Fields. Oxford University Press. https://www.oxfordscholarship.com/view/10.1093/ acprof:oso/9780195058239.001.0001/acprof-9780195058239 
Mayseless, N., Hawthorne, G., \& Reiss, A. L. (2019). Real-life creative problem solving in teams: FNIRS based hyperscanning study. NeuroImage, 203, 116161. https://doi.org/10.1016/j.neuroimage.2019.116161

Nguyen, T. A., \& Zeng, Y. (2014). A physiological study of relationship between designer's mental effort and mental stress during conceptual design. Computer-Aided Design, 54, 3-18. https://doi.org/10.1016/j.cad.2013.10.002

NIRSport. (2015). NIRx Medical Technologies, LLC. https://nirx.net/

Okamoto, M., Dan, H., Shimizu, K., Takeo, K., Amita, T., Oda, I., Konishi, I., Sakamoto, K., Isobe, S., Suzuki, T., Kohyama, K., \& Dan, I. (2004). Multimodal assessment of cortical activation during apple peeling by NIRS and fMRI. NeuroImage, 21(4), 1275-1288. https://doi.org/10.1016/j.neuroimage.2003.12.003

Oostenveld, R., \& Praamstra, P. (2001). The five percent electrode system for high-resolution EEG and ERP measurements. Clinical Neurophysiology, 112(4), 713-719. https://doi.org/10.1016/S1388-2457(00)00527-7

OpenBCI Inc. (2019). OpenBCI Inc. https://openbci.com/

Pinti, P., Tachtsidis, I., Hamilton, A., Hirsch, J., Aichelburg, C., Gilbert, S., \& Burgess, P. W. (2018). The present and future use of functional near-infrared spectroscopy (fNIRS) for cognitive neuroscience. Annals of the New York Academy of Sciences. http://dx.doi.org/10.1111/nyas.13948

Piper, S. K., Krueger, A., Koch, S. P., Mehnert, J., Habermehl, C., Steinbrink, J., Obrig, H., \& Schmitz, C. H. (2014). A Wearable Multi-Channel fNIRS System for Brain Imaging in Freely Moving Subjects. NeuroImage, 85(0 1). https://doi.org/10.1016/j.neuroimage.2013.06.062

Safaie, J., Grebe, R., Moghaddam, H. A., \& Wallois, F. (2013). Toward a fully integrated wireless wearable EEG-NIRS bimodal acquisition system. Journal of Neural Engineering, 10(5), 056001. https://doi.org/10.1088/1741-2560/10/5/056001

Sawan, M., Salam, M. T., Le Lan, J., Kassab, A., Gélinas, S., Vannasing, P., Lesage, F., Lassonde, M., \& Nguyen, D. K. (2013). Wireless Recording Systems: From Noninvasive EEG-NIRS to Invasive EEG Devices. IEEE Transactions on Biomedical Circuits and Systems, 7(2), 186-195. https://doi.org/10.1109/TBCAS.2013.2255595

Shealy, T., Gero, J., Hu, M., \& Milovanovic, J. (2020). Concept generation techniques change patterns of brain activation during engineering design. Design Science, 6. https://doi.org/10.1017/dsj.2020.30

Shin, J., von Lühmann, A., Blankertz, B., Kim, D.-W., Jeong, J., Hwang, H.-J., \& Müller, K.-R. (2017). Open Access Dataset for EEG+NIRS Single-Trial Classification. IEEE Transactions on Neural Systems and Rehabilitation Engineering, 25(10), 1735-1745. https://doi.org/10.1109/TNSRE.2016.2628057

Solovey, E. T., Girouard, A., Chauncey, K., Hirshfield, L. M., Sassaroli, A., Zheng, F., Fantini, S., \& Jacob, R. J. K. (2009). Using fNIRS brain sensing in realistic HCI settings: Experiments and guidelines. 10.

Steinert, M., \& Jablokow, K. (2013). Triangulating front end engineering design activities with physiology data and psychological preferences. 109-118.

Teplan, M. (2002). Fundamentals of EEG Measurement. Measurement Science Review, 2, 11.

The iMotions Platform (8.1). (2020). [Computer software]. iMotions. https://imotions.com/

Ultracortex "Mark IV" EEG Headset. (2019). OpenBCI Inc. https://shop.openbci.com/products/ultracortexmark-iv

Vieira, S., Gero, J. S., Delmoral, J., Gattol, V., Fernandes, C., Parente, M., \& Fernandes, A. A. (2020). The neurophysiological activations of mechanical engineers and industrial designers while designing and problem-solving. Design Science, 6. https://doi.org/10.1017/dsj.2020.26

Vieira, S., Gero, J. S., Delmoral, J., Li, S., Cascini, G., \& Fernandes, A. (2020). Brain activity in constrained and open design spaces: An EEG study. Proceedings of the Sixth International Conference on Design Creativity (ICDC 2020), 068-075.

von Lühmann, A., Herff, C., Heger, D., \& Schultz, T. (2015). Toward a Wireless Open Source Instrument: Functional Near-infrared Spectroscopy in Mobile Neuroergonomics and BCI Applications. Frontiers in Human Neuroscience, 9. https://doi.org/10.3389/fnhum.2015.00617

von Lühmann, A., Wabnitz, H., Sander, T., \& Müller, K.-R. (2017). M3BA: A Mobile, Modular, Multimodal Biosignal Acquisition Architecture for Miniaturized EEG-NIRS-Based Hybrid BCI and Monitoring. IEEE Transactions on Biomedical Engineering, 64(6), 1199-1210. https://doi.org/10.1109/TBME.2016.2594127

Wulvik, A. S., Dybvik, H., \& Steinert, M. (2019). Investigating the relationship between mental state (workload and affect) and physiology in a control room setting (ship bridge simulator). Cognition, Technology \& Work. https://doi.org/10.1007/s10111-019-00553-8

Xu, J., Slagle, J. M., Banerjee, A., Bracken, B., \& Weinger, M. B. (2019). Use of a Portable Functional NearInfrared Spectroscopy (fNIRS) System to Examine Team Experience During Crisis Event Management in Clinical Simulations. Frontiers in Human Neuroscience, 13. https://doi.org/10.3389/fnhum.2019.00085 